

\begin{tabular}{|c|l|}
\hline Title & $\begin{array}{l}\text { Cylindrical microlasers and light emitting } \\
\text { devices from conducting polymers }\end{array}$ \\
\hline Author(s) & Frolov, S. V.; Fujii, A.; Chinn, D. et al. \\
\hline Citation & Applied Physics Letters. 72(22) p. 2811-p. 2813 \\
\hline Issue Date & $1998-05-27$ \\
\hline oaire:version & VoR \\
\hline URL & https://hdl. handle. net/11094/75651 \\
\hline rights & \\
\hline Note & \\
\hline
\end{tabular}

Osaka University Knowledge Archive : OUKA

https://ir. Library. osaka-u. ac. jp/

Osaka University 


\section{Cylindrical microlasers and light emitting devices from conducting polymers}

Cite as: Appl. Phys. Lett. 72, 2811 (1998); https://doi.org/10.1063/1.121466

Submitted: 26 February 1998 . Accepted: 01 April 1998. Published Online: 27 May 1998

S. V. Frolov, A. Fujii, D. Chinn, Z. V. Vardeny, K. Yoshino, and R. V. Gregory

ARTICLES YOU MAY BE INTERESTED IN

Plastic microring lasers on fibers and wires

Applied Physics Letters 72, 1802 (1998); https://doi.org/10.1063/1.121189

High quantum efficiency luminescence from a conducting polymer in solution: A novel polymer laser dye

Applied Physics Letters 60, 3215 (1992); https://doi.org/10.1063/1.106743

Stimulated emission and lasing in dye-doped organic thin films with Forster transfer

Applied Physics Letters 71, 2230 (1997); https://doi.org/10.1063/1.120065






\title{
Cylindrical microlasers and light emitting devices from conducting polymers
}

\author{
S. V. Frolov, ${ }^{\text {a) }}$ A. Fujii, ${ }^{\text {b) }}$ D. Chinn, and Z. V. Vardeny \\ Physics Department, University of Utah, Salt Lake City, Utah 84112 \\ K. Yoshino \\ Electronic Engineering Department, Faculty of Engineering, Osaka University, Yamada-Oka 2-1 Suita, \\ Osaka 565, Japan
}

R. V. Gregory

School of Textiles, Fiber and Polymer Science, Clemson University, Clemson, South Carolina 29634

(Received 26 February 1998; accepted for publication 1 April 1998)

\begin{abstract}
Substantially improved, photopumped polymer lasers are demonstrated using microrings and microdisks of various diameters $D$ ranging from 5 to $200 \mu \mathrm{m}$. Various cavity-dependent laser modes were observed, which for $D<10 \mu \mathrm{m}$ were dominated by a single longitudinal mode with linewidth of less than $1 \AA$. These microlasers were also characterized by $Q$ of order 5000 , low threshold excitation energy of order $100 \mathrm{pJ} /$ pulse for pulse duration ranging from $100 \mathrm{ps}$ to sub- $\mu \mathrm{s}$, and an abrupt increase in the emission directionality and polarization degree. Light emitting diodes with cylindrical geometry, fully compatible with these microlasers are also demonstrated. (C) 1998 American Institute of Physics. [S0003-6951(98)04222-3]
\end{abstract}

Following the discovery of laser action in luminescent conducting polymer thin films, ${ }^{1,2}$ extensive studies of various lasing mechanisms in these materials ${ }^{3-7}$ and other organic solids ${ }^{8,9}$ have been directed toward the realization of electrically driven organic solid-state lasers. Successful fabrication of laser diodes from highly luminescent polymers requires low laser threshold current densities. ${ }^{2}$ Microcavities, in particular, have been shown to produce high quality factor, $Q{ }^{10}$ which may consequently lead to low laser thresholds. Planar microcavities, ${ }^{1}$ however, are prone to losses due to imperfect reflections and emission leakage to the sides of the microcavity plane. Cylindrical microcavities, on the other hand, poses higher $Q$ and have been found very effective for demonstration of lasing in $\pi$-conjugated polymers. ${ }^{6}$ These optical resonators may support wave guided and whispering gallery modes, ${ }^{10}$ both of which are due to total internal reflections inside the active volume and thus can be practically lossless. The resonator optical losses are then determined by selfabsorption and scattering in the gain medium.

Although several different $\pi$-conjugated polymers exhibit optical gain and lasing when photoexcited,,${ }^{1-7}$ we found the internal optical losses in 2,5-dioctyloxy $\operatorname{poly}(p$ phenylene-vinylene) or DOO-PPV [Fig. 1(a), inset], to be particularly low; we estimated the corresponding exponential loss coefficient to be of order $30 \mathrm{~cm}^{-1}$ (Ref. 11). The corresponding self-absorption limited cavity finesse, $Q$, was calculated to be of order $3000 .{ }^{6}$ Using thin DOO-PPV films with typical thickness of $0.5 \mu \mathrm{m}$, we fabricated two types of cylindrical microcavities (see Fig. 1, insets); (i) microrings on optical fibers, metal wires and polymer fibers, and (ii) flat microdisks on glass substrates. The microrings were formed

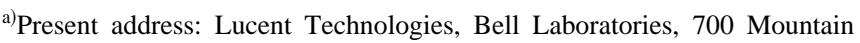
Avenue, Murray Hill, NJ 07974.

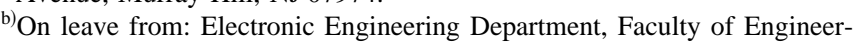
ing, Osaka University, Yamada-Oka 2-1 Suita, Osaka 565, Japan.
}

by coating and subsequent evaporation of the DOO-PPV saturated chloroform solution around cylindrical fibers and wires with diameters ranging from 5 to $200 \mu \mathrm{m}$. The microdisks of various diameters from 4 to $125 \mu \mathrm{m}$ were photolithographycally etched from thin DOO-PPV films, which were uniformly spin coated on flat quartz substrates.

The DOO-PPV microstructures were photoexcited using a frequency doubled (at $532 \mathrm{~nm}$ ) Nd:YAG regenerative laser

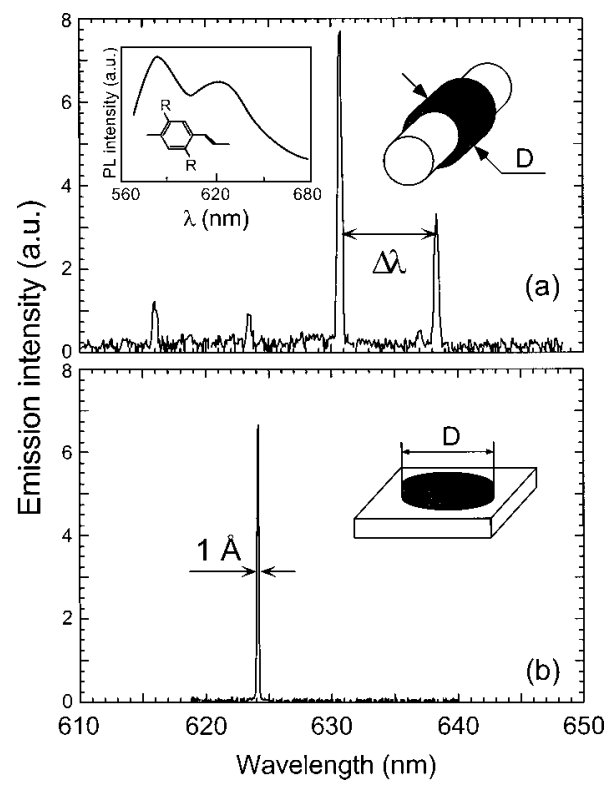

FIG. 1. Emission spectra of DOO-PPV cylindrical microlasers excited with 100 ps pulses at $532 \mathrm{~nm}$, with intensities $P$ above the laser threshold excitation intensity $P_{0}$ of order $100 \mathrm{pJ}$ of absorbed energy/pulse. The insets show schematically the microlaser structures, where $D$ is the outer diameter. (a) A microring with $D=11 \mu \mathrm{m}$; the polymer repeat unit $\left(R=\mathrm{OC}_{8} \mathrm{H}_{17}\right)$ and the PL spectrum for $P<P_{0}$ showing a band width of $\sim 100 \mathrm{~nm}$ are also shown in the inset. (b) A microdisk with $D=8 \mu \mathrm{m}$, showing a single longitudinal laser mode with the linewidth limited by the spectrometer resolution. 
amplifier producing either $100 \mathrm{ps}, 10 \mathrm{~ns}$, or $200 \mathrm{~ns}$ pulses at $1 \mathrm{kHz}$ repetition rate. Individual microrings of approximately $100 \mu \mathrm{m}$ in lateral length were photoexcited by focusing the pump beam with a cylindrical lens, whereas single microdisks could be photopumped using a round lens focusing the pump laser beam into an excitation area of $30 \mu \mathrm{m}$ in diameter. The polymer microcavity emission was collected by a round lens in the plane of the microring, or microdisk, and spectrally analyzed using a $0.6 \mathrm{~m}$ spectrometer with spectral resolution of $1 \AA$. All measurements were conducted in a dynamic vacuum to avoid excessive photo-oxidation, allowing the polymer microcavities to continuously lase for several hours without substantial degradation.

In general, microcavity resonances can drastically change the spontaneous emission spectrum of the active gain medium via the quantum electrodynamic effect. ${ }^{1,10}$ In our case, however, because the characteristic sizes of the DOOPPV microcavities are sufficiently larger than the emission wavelength $\lambda$, the microcavities photoluminescence (PL) band for excitation intensities, $P$, below lasing threshold is not affected by the cavity resonances and remains similar to the PL spectrum of free standing films with typical spectral linewidth of $\sim 100 \mathrm{~nm}$ (Ref. 4) [Fig. 1(a), inset]. Dramatic changes occur in the microcavity emission spectrum when $P$ exceeds the laser threshold excitation intensity, $P_{0}$; in this case the emission spectrum collapses into several dominant microcavity modes. ${ }^{6}$ In microring cavities, the thin polymer film deposited around the optical fiber supports wave guided modes with resonant wavelengths, $\lambda_{m}$, separated by $\Delta \lambda$ $=\lambda_{m}-\lambda_{m+1}$, as given by ${ }^{12}$

$$
\Delta \lambda=\lambda^{2} / \pi D n_{\text {eff }},
$$

where $m$ is an integer (mode number) and $n_{\text {eff }}$ is the effective refraction index of the curved polymer film. Figure 1(a) shows the multimode spectrum of a DOO-PPV microring laser at $P>P_{0}$ with an outer diameter, $D$, of $\sim 11 \mu \mathrm{m}$, excited with 100 ps pulses. $P_{0}$ for this microlaser, expressed in terms of total absorbed energy per pulse is only $0.1 \mathrm{~nJ}$. We found that Eq. (1) with $n_{\text {eff }} \sim 1.7$ (Ref. 11) agrees very well with the observed mode structure and adequately describes the spectra of all microring lasers with $10 \mu \mathrm{m}<D$ $<200 \mu \mathrm{m}$.

Similar results were obtained with DOO-PPV microdisks. We found that $\Delta \lambda$ for the microdisk laser is also given by Eq. (1), where $n_{\text {eff }}$ refers to the effective refraction index of a microdisk. Equation (1) shows that $\Delta \lambda$ increases as $D$ decreases. Since the spectral width, $\Gamma$, of the optical gain in DOO-PPV is on the order of $10 \mathrm{~nm}$ (Ref. 4) and independent of $D$, then the single longitudinal mode lasing takes place when $\Delta \lambda>\Gamma$. Indeed, we found that for $D<10 \mu \mathrm{m}$ both microring and microdisk lasers operate in the single mode regime. This is illustrated in Fig. 1(b), where the spectrum of a single mode microdisk laser $(D=8 \mu \mathrm{m})$ is shown. We note that $P_{0}$ of this microdisk is similar to that of the same diameter microring, and is of order $100 \mathrm{pJ} / \mathrm{pulse}$.

Since the DOO-PPV microlasers are characterized by fairly low $P_{0}$ and high PL quantum efficiency, we could also achieve laser action with minimal heating using $10 \mathrm{~ns}$ and $200 \mathrm{~ns}$ pulses, respectively, significantly longer than the 100 ps pulses used previously. ${ }^{4,6}$ The successful photopumped lasing of $\pi$-conjugated polymer microcavities with sub- $\mu \mathrm{s}$ pulses is a significant advance towards electrically pumped organic lasers, which should be operated with relatively short electrical pulses. We estimate the microlasers $Q$ value to be about 5000, which in addition to the low laser threshold, results in narrow laser emission lines. In fact, the spectral linewidth of the microlasers shown in Fig. 1 is limited by the spectrometer resolution, and thus estimated to be no broader than $1 \AA$. To our knowledge, these microlasers exhibit the narrowest linewidth achieved so far in the field of solid state polymer lasers. It is worth noting that for the single mode microlaser shown in Fig. 1(b), the broad DOO-PPV PL band of $\sim 100 \mathrm{~nm}$ at $P<P_{0}$ collapses for $P>P_{0}$ into a narrow laser line of less than $1 \AA$. This amounts to spectral narrowing of about 1000, which represents an improvement by almost two orders of magnitude compared to the values previously reported in the literature ${ }^{1-5}$ for other organic laser action devices.

The laser emission collected from the microlasers is caused by tunneling of evanescent EM waves through the outer film surface and light scattering at imperfections. ${ }^{10} \mathrm{We}$ found that the intensity distribution in the microlaser emission is concentrated in a small cone of 60 miliradians in the plane of the microcavity, evenly spread around the microcavity principal axis. ${ }^{6}$ Simultaneously, for $P>P_{0}$ this emission is linearly polarized along the microcavity cylindrical axis with polarization ratio of more than 100:1. For the microring lasers, it is also possible to couple the laser emission directly into the fiber by using a tilted, builtin fiber Bragg grating. ${ }^{13}$ Similar coupling techniques may be used for microdisk lasers, where the emission could be coupled into closely aligned channel wave guides. ${ }^{14}$

In the next development phase towards polymer laserdiodes, we show that the cylindrical microcavities can simultaneously operate as photopumped microlasers and electrically driven light emitting devices (LEDs). In case of microring devices, we deposited the DOO-PPV film around a thin gold wire (Fig. 2) forming a microring with $D$ $=30 \mu \mathrm{m}$. The laser mode spectrum of this microring at $P$ $>P_{0}$ is shown in Fig. 2(a). Being close to the metallic interface, wave guided modes in thin polymer films experience large absorption losses. Therefore, lasing occurs only in sufficiently thick films via whispering gallery modes confined to the outer rim of the microring; the inner surface may be metallic or made from other conducting materials. In Fig. 2(b) we demonstrate the laser modes of a DOO-PPV microring deposited around a polyaniline (PANI) fiber with $D$ $=80 \mu \mathrm{m}$. Since doped PANI is a conducting polymer with high electrical conductivity, this example shows the possibility of an all-organic laser diode. We note that when capped with $\mathrm{Al}$ electrodes [Fig. 3(a)], these microring devices can work as cylindrical LEDs with a typical current versus forward bias voltage $(I-V)$ characteristic shown in Fig. 3(b).

In case of microdisk devices, we deposited DOO-PPV thin film on good quality, indium tin oxide (ITO) substrates for hole injection, capped with an $\mathrm{Al}$ layer for electron injection, and then etched both the polymer film and Al layer to produce patterned microdisks of various $D$. The $I-V$ and electroluminescence intensity (EL) versus $V$ characteristics at forward biasing of a microdisk LED with $D=125 \mu \mathrm{m}$ are 




FIG. 2. Emission spectra of microring lasers composed of $\sim 3 \mu \mathrm{m}$ thick DOO-PPV films wrapped around a gold wire (a) and a polyaniline (PANI) polymer fiber (b), showing whispering gallery laser modes with separation $\Delta \lambda$ given by Eq. (1). The excitation conditions and spectral resolution are as in Fig. 1 with $P_{0}$ of $\sim 1 \mathrm{~nJ} /$ pulse. The insets show schematically the microstructure cross sections and their outer diameter $D$.

shown in Fig. 3(b). Its EL spectrum measured at $V=30 \mathrm{~V}$ is identical to the PL spectrum shown in Fig. 1(a). We emphasize that the same devices produce well-resolved photopumped laser modes for $P>P_{0}$, similar to those in Fig. 2.

The micro LEDs were characterized using DC currents. However, in order to achieve a current-driven organic laser, it will be necessary to use pulsed current sources. We have determined that laser action is possible with sub- $\mu$ s light

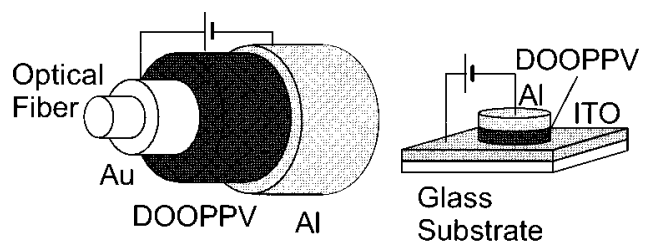

(a)

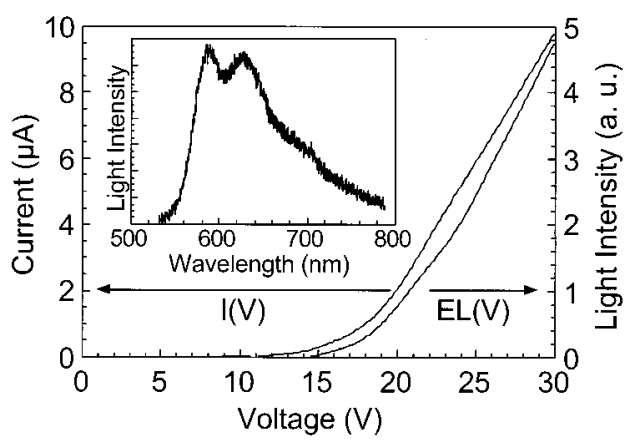

(b)

FIG. 3. (a) Schematic structures of multilayered microring (left) and microdisk (right) LEDs. The microring structure is composed of a gold wire for hole injection, followed by an active DOO-PPV film and a thin, semitransparent aluminum film for electron injection. The microdisk may be composed of the same layers, but instead of gold, it is deposited on a transparent ITO film for hole injection. (b) The characteristic current vs forward applied bias voltage $(I-V)$ and electroluminescence vs bias voltage (EL- $V)$ for a microdisk LED with $D=125 \mu \mathrm{m}$. The inset shows the EL spectrum of the microdisk LED obtained at $V=30 \mathrm{~V}$. pulses. We can assume that sub- $\mu$ s electrical pulses may be sufficiently short for electrically driven polymer laser diodes. From $P_{0}$ of our photopumped microlasers and assuming a $5 \%$ internal quantum efficiency, we estimate the threshold current density necessary for lasing to be of order 5 $\times 10^{3} \mathrm{~A} / \mathrm{cm}^{2}$. Such current densities are within the present limits of polymer LEDs, where current densities as high as $10^{6} \mathrm{~A} / \mathrm{cm}^{2}$ have recently been demonstrated. ${ }^{15}$

In conclusion, we have developed cylindrical photopumped microlasers based on $\pi$-conjugated polymers characterized by high $Q$, narrow spectral lines and low laser threshold excitation intensities. These characteristics have been improved by two orders of magnitude compared to the previously reported values for polymer lasers. We have shown that the developed microlasers are fully compatible with the present polymer LED technology and allow submicrosecond excitation. We have also demonstrated electrically driven cylindrical microring and microdisk LEDs, which can simultaneously operate as photopumped microlasers. These findings reveal a promising route toward electrically driven polymer laser diodes.

The authors thank W. Gellermann and M. Shkunov for technical assistance and C. Williams for help in preparation of thin optical fibers. This work was supported in part by the DOE and the NEDO foundation.

${ }^{1}$ N. Tessler, G. J. Denton, and R. H. Friend, Nature (London) 382, 695 (1996).

${ }^{2}$ F. Hide, M. A. Diaz-Garcia, B. J. Schwartz, M. R. Andersson, Q. Pei, and A. J. Heeger, Chem. Phys. Lett. 256, 424 (1996); Science 273, 1833 (1996).

${ }^{3}$ H. J. Brouwer, V. V. Krasnikov, A. Hilberer, and G. Hadziioannou, Adv. Mater. 8, 935 (1996); G. Gelink, J. W. Warman, M. Remmers, and D. Neher, Chem. Phys. Lett. 265, 320 (1997).

${ }^{4}$ S. V. Frolov, M. Ozaki, W. Gellermann, K. Yoshino, and Z. V. Vardeny, Phys. Rev. Lett. 78, 729 (1997).

${ }^{5}$ X. Long, A. Malinowski, D. D. C. Bradley, M. Inbasekaran, and E. P. Woo, Chem. Phys. Lett. 272, 6 (1997).

${ }^{6}$ S. V. Frolov, M. Shkunov, Z. V. Vardeny, and K. Yoshino, Phys. Rev. B 56, R4363 (1997).

${ }^{7}$ G. J. Denton, N. Tessler, M. A. Stevens, and R. H. Friend, Adv. Mater. 9, 547 (1997).

${ }^{8}$ V. G. Kozlov, V. Bulovic, P. E. Burrows, and S. R. Forrest, Nature (London) 389, 362 (1997).

${ }^{9}$ M. Berggren, A. Dodabalapur, R. E. Slusher, and Z. Bao, Nature (London) 389, 466 (1997); Appl. Phys. Lett. 71, 2230 (1997); Adv. Mater. 9, 968 (1997).

${ }^{10}$ Y. Yamamoto and R. Slusher, Phys. Today 46, 66 (1993); Microcavities and Photonic Band Gaps: Physics and Applications, edited by J. Rarity and C. Weisbuch (NATO, 1996).

${ }^{11}$ S. V. Frolov, Z. V. Vardeny, and K. Yoshino, Phys. Rev. B 57, 9141 (1998).

${ }^{12}$ H. P. Weber and R. Ulrich, Appl. Phys. Lett. 19, 38 (1971); M. KuwataGonokami, R. H. Jordan, A. Dodabalapur, H. Katz, M. L. Schiling, and R. E. Slusher, Opt. Lett. 20, 2093 (1995).

${ }^{13}$ T. Erdogan and J. E. Sipe, J. Opt. Soc. Am. A 13, 296 (1996).

${ }^{14} \mathrm{H}$. Kogelnik, in Guided-Wave Optoelectronics, edited by T. Tamir (Springer, Berlin, 1988).

${ }^{15}$ D. G. Lindzey, D. D. C. Bradley, S. F. Alvarado, and P. F. Seidler, Nature (London) 386, 135 (1997). 